\title{
Multi-colour light variation of AGB stars observed with ISO ${ }^{\star, \star \star}$
}

\author{
F. Kerschbaum ${ }^{1}$, T. Lebzelter ${ }^{1}$, and C. Lazaro ${ }^{2}$ \\ 1 Institut für Astronomie, Türkenschanzstraße 17, 1180 Wien, Austria \\ 2 Instituto de Astrofísica de Canarias, and Departamento de Astrofísica, Facultad de Física, \\ Universidad de La Laguna, 38271 La Laguna, Tenerife, Spain
}

Received 12 March 2001 / Accepted 8 June 2001

\begin{abstract}
New visual light curves and infrared multi-epoch photometry are presented for a sample of AGBstars spectroscopically observed with ISO. While the ISO work is or will be presented elsewhere, the aim of this paper is to give an overview of the properties of the light change of the objects. This information is crucial for the interpretation of the valuable ISO material. Using the University of Vienna Twin Automatic Photoelectric Telescope (APT) we monitored the stars of our sample in the photometric bands $V$ and $I_{\mathrm{C}}$. We present the light curve of each object and derive the parameters of the current light change, such as period(s) and amplitude. Furthermore we give $V-I_{\mathrm{C}}$ colours and colour variations for these objects. Our results allow us to derive some general results on semiregular and irregular variables. Only in less than $50 \%$ of the cases could we confirm the GCVS period. Moreover, we did not find any pronounced difference between SRb and Lb variables in the regularity of the light curve. The existence of rapid oscillations indicated by Hipparcos data could not be confirmed. In addition to the visual light changes we present new near infrared photometry data. Although typically only few data points are available, they can be viewed relative to the better-monitored visual light curves providing information on possible phase shifts and differences in amplitude in different parts of the spectrum. Furthermore, multi-epoch photometry allows us to derive mean colours for these objects.
\end{abstract}

Key words. stars: variables: general - stars: AGB and post AGB - infrared: stars - techniques: photometric space vehicles

\section{Introduction}

Pulsation is a critical aspect of the late stages of stellar evolution. The longest known indication of these pulsations are the well-expressed regular and irregular variations in brightness of objects on the Asymptotic Giant Branch (AGB) like Mira-, Semiregular (SRV) and Irregular Variables (IRV). This pulsation affects the structure of the stellar atmosphere. As it drives the extension of the outer stellar layers it produces an environment for the formation of dust, which is an important factor for the mass loss and therefore for the stellar evolution.

The theoretical description of the pulsational phenomena in the atmosphere of AGB-stars is now making considerable progress (e.g. Höfner et al. 1998; Höfner 1999; Winters et al. 2000). New observational techniques like

Send offprint requests to: F. Kerschbaum, e-mail: Franz.Kerschbaum@univie.ac.at

* Based on observations made with the Carlos Sánchez Telescope operated on the island of Tenerife by the Instituto de Astrofísica de Canarias (IAC) in the Observatorio del Teide, Izaña.

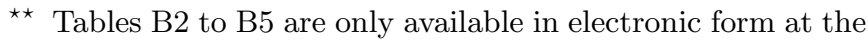
CDS via anonymous ftp to

cdsarc.u-strasbg.fr $(130.79 .128 .5)$ or via

http://cdsweb.u-strasbg.fr/cgi-bin/qcat?J/A+A/375/527 the ISO-satellite provide important input parameters for the models. Still, it is only possible to obtain an overall picture if fundamental parameters like the period and the regularity of the light changes are taken into account. An interpretation of spectroscopic observations needs the relation of the data to the overall pulsational variations visible as light changes. Differences in the variations in different parts of the spectrum help us to understand the influence of the pulsation on the atmospheric structure. While the main mechanisms and effects of periodic variability are now understood, irregular changes observed in the light curves of many of these stars still lack a general explanation.

For an understanding of these irregular variations, detailed observational material is very much needed. The light changes of AGB variables typically occur with periods between 30 and 600 days. This makes it very difficult to obtain reliable light curves, especially for semiregular variables. Hence, for only a very small number of objects are good, long and well-sampled light curves published. Even the most important pulsational parameters like period or light amplitude found in the literature have to be checked in detail and are questionable in many cases, as shown by Lebzelter et al. (1995). 
However, the analysis of the light change of long period variables has made considerable progress in recent years, mainly due to two different approaches: On the one hand, the archives of light measurements provided by amateur astronomers have been transformed to electronic form so that these data are now available in a more convenient way. Several investigations of AGB variables are based on these data (e.g. Mattei et al. 1997; Kiss et al. 1999, 2000). The importance of these light curves lies in their sometimes extremely long time coverage, so that, despite the inaccuracies of the individual measurements, typical periods, especially long time variations, could be derived. Multi-periodicity has been found in several AGB variables in this way. On the other hand, long time-scale photoelectric light curves, provided either by amateur astronomers (Percy et al. 1996) or by automatic telescopes (e.g. Percy $\&$ Au 1994; Christian et al. 1995), give new insight into the variability of short period and small amplitude variables, and also into the short time-scale variability as a contribution to the light change of large-amplitude AGB stars.

A program to monitor semiregular and irregular variables at high photometric accuracy and with a good time sampling has been started at the University of Vienna to provide high-quality light curves necessary for a detailed study of the irregular behaviour in the light change. First results have already been presented elsewhere (Lebzelter 1999). In the present paper we show and discuss the light curves of a sample of semiregular and irregular variables, which have been investigated using the ISO satellite (e.g. Hron et al. 1998; Posch et al. 1999; Aringer et al. 1999; Jørgensen et al. 2000).

Since the visual light changes are dominated by the opacity effects of a few temperature-sensitive molecules, the physically more relevant luminosity changes can only be investigated in the infrared, where most of the stellar radiation originates. Therefore, we also present in this paper new multi-epoch near infrared photometry of some of the objects monitored in the optical range and of a few other AGB-variables observed within our ISO programs. For several objects in our sample this is the first measurement of their infrared light change. Without being able to derive the mechanism of irregular light change in these stars, this paper - with its optical and near infrared photometry and its connection to further stellar parameters derived from the ISO spectra - provides a unique dataset for investigating this question and sets limits on possible explanations.

\section{Observations}

\subsection{The sample}

In the course of the open-time ISO programmes fkerschb/orichsrv and /orichsrl as well as jhron/varlpv and $/$ varlpv2 and finally the solicited proposal for the extended mission fkerschb/zzagb2pn, a large sample of AGB variables of Mira, semiregular and irregular type was observed with the short wave spectrometer in order to compare these infrared spectra with state of the art theoretical models and to use the results to investigate the interrelation of pulsational behaviour, atmospheric structure and mass loss of long-period variables.

Whereas the programmes with obsid: fkerschb try to study a large number of different objects with a single observation at a random pulsational phase, those by jhron concentrate on fewer objects but carry out multi-epoch spectroscopic observations in order to cover a good fraction of the pulsational phases. These two complementary approaches both need supplementary observations from the ground in order to characterise the pulsational behaviour of the stars and to estimate the exact phases when the spectra were taken.

Consequently, we tried to start observational monitoring programmes in both the visual and the near infrared range for as many objects as possible. Because of a number of limiting factors only a relatively small sub-sample of our ISO objects could finally be monitored quasi-simultaneously with the ISO observations.

Moreover, in the course of the near infrared observations (see below) a small number of additional Semiregular, Irregular and Mira variables were observed. These are also included in the tables for reference.

\subsection{Photo-electric photometry}

The variability of many late-type stars has been monitored for a long time by visual measurements. Following the light change of these stars with photoelectric precision has recently become more efficient with the use of automatic telescopes. This increase in photometric accuracy and time resolution opened new possibilities for studying the complex and small amplitude variability of these objects.

Since 1996 the University of Vienna has owned two automatic photoelectric telescopes (APT) at Washington Camp in Arizona, named Wolfgang and Amadeus. The two APTs are optimised for the blue and red wavelength region, respectively. Both telescopes have $0.75 \mathrm{~m}$ primary mirrors. A detailed description of Wolfgang and Amadeus is given in Strassmeier et al. (1997). Because of the frequent summer thunderstorms in Arizona, Wolfgang and Amadeus are closed during July and August.

Typically one data point per night was obtained with the $V, R_{\mathrm{C}}$ and $I_{\mathrm{C}}$ filters. The filter specifications can be found in Strassmeier et al. (1997). We used only data with a maximum error of 0.02 (standard deviation of three single measurements), i.e. the measurements were generally better than $1 \%$ of the mean amplitude. It is therefore possible to detect and analyse even the fine structure of the light variations.

The star-finding procedure of the APTs requires an estimate of the star's current brightness (see Strassmeier et al. 1997 for further details on the APT finding procedure). While a variability with amplitudes of up to 1.5 or $2 \mathrm{mag}$, i.e. a deviation of the true brightness from the 
mean brightness by $1 \mathrm{mag}$, still allows us to find the star, large amplitude variables like Miras with a significantly more extended light change cannot be handled by the APT finding procedure. Therefore only SRVs and IRVs could be observed with the APT.

Absolute photometry was used to calculate the brightness of the comparison stars which are listed in Table A.1. The brightness and light change of the programme stars was then derived relative to these comparison stars (differential photometry). Note that due to the lack of photometric standards at large $V-I_{\mathrm{C}}$ values our program stars are in a different colour range than the comparison stars we used. Comparison stars of later spectral type all turned out to be variable (e.g. Lebzelter 1999). While our absolute values may therefore have an additional uncertainty, the variations in light and colour discussed in this paper should not be affected.

Previous to this program we obtained some data points for g Her with the Phoenix 10 automatic telescope (Boyd et al. 1984).

\subsection{Near infrared photometry}

At the Observatorio del Teide, the $1.5 \mathrm{~m}$ "Carlos Sánchez Telescope" (CST) of the Instituto de Astrofísica de Canarias (IAC) on Izaña, Tenerife, was used with the "CVF Photometer-Spectrophotometer" in order to derive $J H K L$-Photometry. The $L$-filter is actually $L^{\prime}(3.78 \mu \mathrm{m})$. The observations were done by FK, CL or in the course of service observations. A few additional observations were kindly provided by Mathias Schultheis, IAP. All observations are listed in Tables B.2 to B.5. For completeness a few already published (Kerschbaum \& Hron 1994, code SRIIa; Kerschbaum 1995, code SRIIb; Kerschbaum et al. 1996, code LBI) observations are also listed. These old data sets which were collected with the same instrumentation using the same standard star lists are indicated by the above codes in column "pub.". In contrast to independent literature data also available for some objects, these data sets can be combined with the new material without any risk of small systematic differences between the photometric systems quite common in the near infrared. Nevertheless we would like to mention - only as examples - the much more complete lightcurves collected for some of our objects by Bagnulo (1999) (R Cas, V Cyg, R For, and SV Peg), the long term sets on R For by Le Bertre (1992) and by Whitelock et al. (1997) or the extensive survey by Catchpole et al. (1979) and the recent compilation of Whitelock et al. (2000).

All observed sources were searched for at their GCVS4 positions, mostly in the $K$-filter. A diaphragm of $15^{\prime \prime}$ was used; beam-switching was done in the East-West direction generally with a throw of 20 ". Standard and programme stars were observed at similar air mass ranges to avoid the non-linear effects of atmospheric extinction in the infrared. The $J H K L^{\prime} M$-photometry was calibrated on the Koornneef (1983a, 1983b) system.

\section{Results}

\subsection{Visual light change from APT}

All stars in our APT sample show an irregular behaviour in their light change, both in the cycle length and in the amplitude of the variation. The accuracy and time resolution of the APT allows us to detect even small amplitude and short period variability. In all of our stars the variations appear to occur on more than one time scale. While a single, fixed period could not be found for any of the stars in our sample, we tried to derive the dominant time scales for each star. Note that these values represent the time scales at the time of the observation. It is possible that these time scales change abrupt or continuously. Although the time span covered with our APT observation is considerably longer than most of the published photoelectric light curves of these stars, we observed less than 10 light cycles for each object. However, the light curve data presented in this paper have been obtained with the aim of characterising the current behaviour of the stars in order to relate it to ISO observations.

In Table 1 we give the quality of the light curve obtained according to the criteria established for semiregular variables by Lebzelter et al. (1995) reaching from quality class Q0 for the worst quality to Q4 for the highest quality. The presented light curves and the resulting quality classes illustrate the difficulty of obtaining high-quality light curves for these stars, i.e. light curves that allow a reliable estimate of the parameters of the variability.

\subsubsection{Period finding}

As noted already in Lebzelter (1999), classical methods for deriving a period, like Fourier analysis (Sperl 1998) alone do not always provide satisfying results for high quality light curves of semiregular and irregular variables. We therefore combined it with visual inspection (maxima, minima) and least square fitting techniques (Sperl 1998). We note that Kiss et al. (1999) successfully used Fourier analysis to derive periods for several SRVs. The dataset they used spanned much longer time scales at much lower photometric accuracy than our observations and included more regular SRa variables. Still, they note that they had problems in deriving a period in this way for several SRVs of their sample as well.

To illustrate the extraordinary behaviour of these stars we plotted the $V$ light curves of all stars of our APT sample in Figs. 1 and 2. In order to allow a direct comparison of amplitude and period of the variation we used the same scaling for all stars of our sample.

The formal frequency uncertainty $\Delta f$ from Fourier analysis is indirectly proportional to the sample length $\Delta T$. A practical rule of thumb $\Delta f=1.5 / \Delta T$ comes from Loumos \& Deeming (1978) which corresponds for our data sets ( $\Delta T$ typically 1000 days) in the period domain to $\Delta P$ of about $4,15,34,69,135$, and 375 days for periods of 50 , $100,150,200,300$, and 500 days, respectively. These very 
Table 1. Variability data of the APT sample. Column 5 gives the wavelength range where the amplitude listed in the GCVS has been measured (according to GCVS nomenclature). The last column gives the quality class of the light curve according to Lebzelter et al. (1995).

\begin{tabular}{|c|c|c|c|c|c|c|c|}
\hline \multirow[t]{2}{*}{ Object } & \multicolumn{4}{|c|}{ GCVS data } & & \multirow{2}{*}{$\begin{array}{l}\text { Quality } \\
\text { class }\end{array}$} \\
\hline & type & $\begin{array}{l}\text { period } \\
{[\mathrm{d}]}\end{array}$ & $\begin{array}{l}\text { ampl. } \\
{[\mathrm{mag}]}\end{array}$ & band & $\begin{array}{l}\text { timescale(s) }[\mathrm{d}] \\
\text { short }\end{array}$ & long & \\
\hline VY Cas & $\mathrm{SRb}$ & 100 & 1.5 & $P$ & 116 (f1), 58 (2f1), 146 & 788 & Q3 \\
\hline RV Cam & $\mathrm{SRb}$ & 101 & 1.3 & $P$ & 177 (f1), 109 (2f2), 132, 218 (f2) & 959 (f1-f2) & Q3 \\
\hline $\mathrm{CW}$ Cnc & $\mathrm{Lb}$ & - & 1.2 & $P$ & $\mathbf{9 7}(\mathrm{f} 1), \mathbf{1 0 0}(\mathrm{f} 2), 49(2 \mathrm{f} 1), 50(2 \mathrm{f} 2)$ & & Q1b \\
\hline RW Vir & $\mathrm{Lb}$ & - & 0.7 & $V$ & $72,116,201$ & 1060 & Q3 \\
\hline Y UMa & $\mathrm{SRb}$ & 168 & 2.1 & $V$ & 166 (2f1), 260, 331 (f1), 358 & 725 & Q1b \\
\hline RX Boo & $\mathrm{SRb}$ & 340 & 2.7 & $P$ & 140 (f1), $70(2 \mathrm{f} 1)$ & & Q1b \\
\hline g Her & $\mathrm{SRb}$ & 89 & 2.0 & $V$ & $\mathbf{8 9}, 74,103$ & 1239 & Q3 \\
\hline AX Sco & $\mathrm{SRb}$ & 138 & 2.2 & $B$ & $\mathbf{1 2 4}, \mathbf{1 2 8}(\mathrm{f} 1), 39,64(2 \mathrm{f} 1)$ & & Q1b \\
\hline V438 Oph & $\mathrm{SRb}$ & 170 & 2.3 & $P$ & $\mathbf{1 5 4}, 129,202$ & 1018 & Q3 \\
\hline TY Dra & $\mathrm{Lb}$ & - & 1.1 & $V$ & $\mathbf{8 9}, 107,410(2 \mathrm{f} 1)$ & $820(\mathrm{f} 1)$ & Q3 \\
\hline T Sge & $\mathrm{SRb}$ & 166 & 1.5 & $P$ & $\mathbf{1 7 8}, \mathbf{3 1 6}, 112$ (10f1) & $1124(\mathrm{f} 1)$ & Q3 \\
\hline V584 Aql & $\mathrm{Lb}$ & - & 1.4 & $P$ & $\mathbf{1 4 3}, 123$ & & Q1b \\
\hline SV Peg & $\mathrm{SRb}$ & 145 & 1.8 & $P$ & 145,220 & & Q1b \\
\hline
\end{tabular}

conservative values are considerably improved when least square fitting techniques are carried out with high quality data sets like ours, but one should keep in mind this principal limitation, which can only be overcome by a longer time base. Another limitation is the density of the sampling which is responsible for the high frequency i.e. short period cut-off. For our sampling a typical Nyquist cut-off is found around 2 days in period, well below the expected periods of long period variables. It even should easily allow the detection of rapid variations ( $<10$ days!) proposed recently by Koen \& Laney (2000) for some M-giants having Hipparcos photometry (see below).

Table 1 gives an overview of the resulting time scales. Table 2 gives results of amplitudes and mean colours. Most stars show variability on short and long time scales. Both of them are listed in Table 1 . The short time scale variability may in some cases be a range of periods, if the star's irregularities and the length of our light curve do not allow a unique determination of the period. Dependent periods (typically differing by a factor of 2 or 10, or being a result of beating) are named in brackets (e.g. f1, 2f1, 10f1, f1f2). The most "important" time scales are shown in bold face. Period lengths of the long time variability are naturally limited by the total time of observation, i.e. about 1200 days. If the period length given in Col. 7 of Table 1 is of that size, it may be that we did not cover the whole light cycle and that the true period is longer than that.

To check the reality and importance of the periods found by our analysis of the data, we present our attempt to fit the observed light curves in Figs. 1 and 2. For the fits shown we used the periods given in the discussion of the individual objects below. Typically, several but not all features of the different light curves could be fitted. Depending on the total number of data points the inclu-
Table 2. Photometric data of the APT sample.

\begin{tabular}{l|rrrrr}
\hline Object & \multicolumn{1}{|c}{$V$ ampl. } & \multicolumn{3}{c}{$V-R_{\mathrm{C}}$} & \multicolumn{3}{c}{$V-I_{\mathrm{C}}$} \\
& \multicolumn{1}{c}{$[\mathrm{mag}]$} & mean & $\max$ & $\min$ & mean \\
\hline VY Cas & $0.6-0.9$ & 1.75 & 4.25 & 3.95 & 4.11 \\
RV Cam & $0.3-1.1$ & 1.87 & 3.96 & 3.44 & 3.80 \\
CW Cnc & $0.3-0.5$ & - & 4.08 & 3.87 & 4.01 \\
RW Vir & $0.2-0.5$ & 1.46 & 3.78 & 3.41 & 3.57 \\
Y UMa & $0.3-0.9$ & 1.89 & 4.49 & 4.17 & 4.38 \\
RX Boo & 0.4 & - & 4.46 & 4.30 & 4.35 \\
g Her & $0.3-0.7$ & 1.35 & 3.65 & 3.31 & 3.48 \\
AX Sco & $0.1-0.8$ & 1.66 & 4.19 & 3.73 & 4.00 \\
V438 Oph & $0.4-1.0$ & 1.80 & 4.35 & 4.02 & 4.24 \\
TY Dra & $0.2-0.9$ & 1.70 & 4.18 & 3.64 & 4.04 \\
T Sge & $0.4-1.2$ & 2.06 & 4.76 & 4.43 & 4.64 \\
V584 Aql & 0.8 & 1.84 & 4.4 & 4.11 & 4.27 \\
SV Peg & $0.2-0.4$ & 1.97 & 4.52 & 4.30 & 4.44 \\
\hline
\end{tabular}

sion of more periods could improve the fits but the uniqueness of such solutions is not given.

\subsection{2. $V-I_{C}$}

As mentioned above, the light change has been monitored not only in $V$ but also in Cousins $I$. We present the result as maximum and minimum $V-I_{\mathrm{C}}$ values for each star in Table 2. $V-I_{\mathrm{C}}$ colours vary by 0.2 to $0.55 \mathrm{mag}$ for the stars in our sample. No phase shift was found between the light change in $V$ and $I_{\mathrm{C}}$ (see Fig. 3 for an example).

Furthermore, we give a mean $V-I_{\mathrm{C}}$ calculated by averaging over the whole dataset of each object. For a part of the sample we obtained also mean $V-R_{\mathrm{C}}$ colours which are also given in Table 2 . T Sge is the star with the largest 


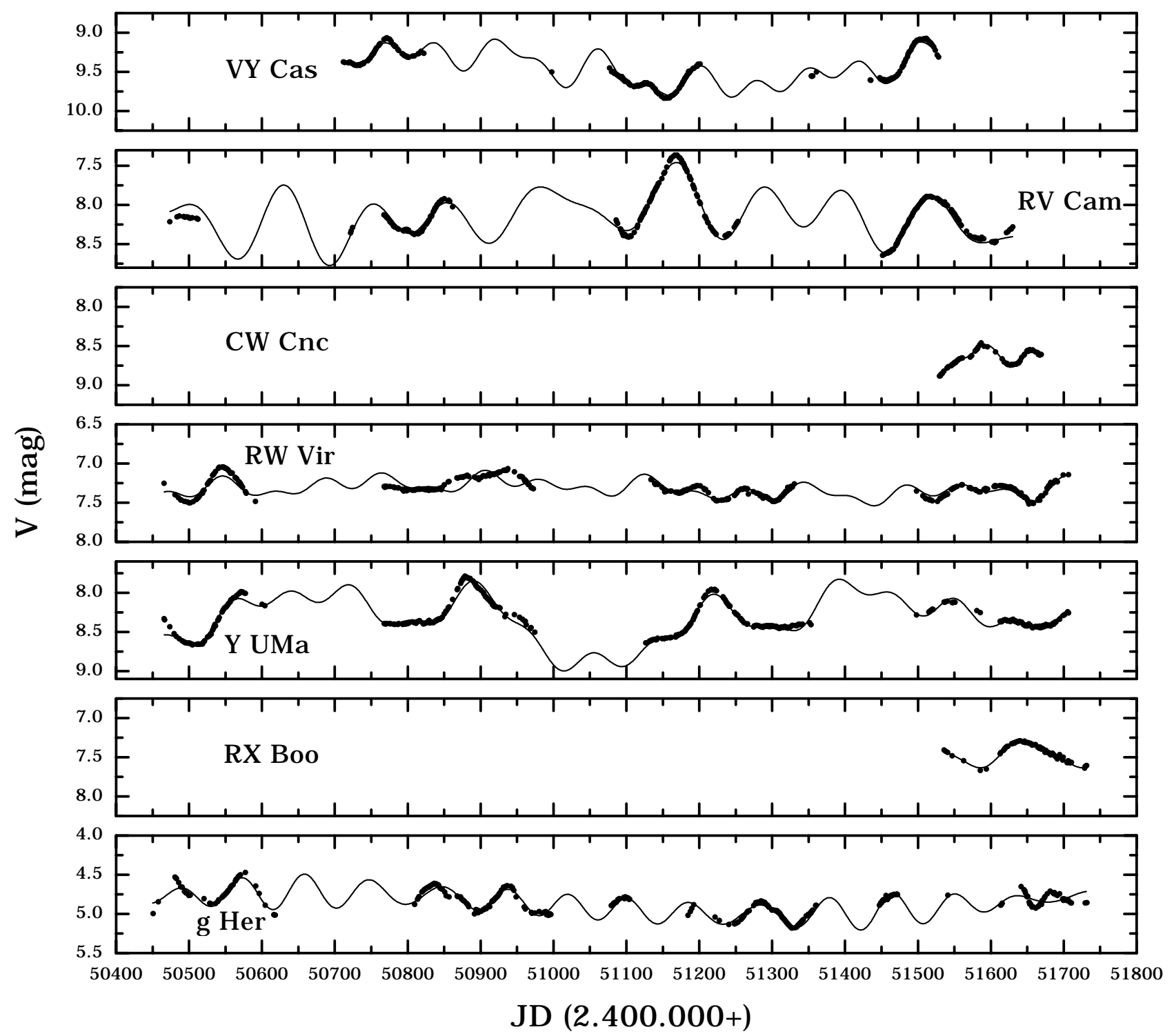

Fig. 1. Compilation of all $V$-lightcurves, Part 1.

mean $V-I_{\mathrm{C}}$ value (4.64), g Her the one with the smallest (3.48). It is interesting to note that $\mathrm{g}$ Her has a somewhat late GCVS spectral type of M6 for its mean $V-I_{\mathrm{C}}$ value. On the other hand V438 Oph and T Sge have mean $V-I_{\mathrm{C}}$ values (4.2 and $4.6 \mathrm{mag}$, respectively) more on the red side of the spectral type ranges given in the GCVS (M0-M7E, M4-M6.5). Both long term changes as well as problematic but common single epoch spectral classification can be responsible for these discrepancies.

Figure 3 shows both the $V$-light curve and the $V-I_{\mathrm{C}}$ variation for the irregular variable TYDra. It can be seen that the change in colour only roughly resembles the visual light curve. The strong brightness maximum at JD 2451100 is accompanied by an outstanding maximum in the $V-I_{\mathrm{C}}$ curve, while other light maxima and minima coincide with a much smaller change in colour. Such differences between the light change in $V$ and $I_{\mathrm{C}}$ have also been found for several other objects of our sample.
An interpretation of this phenomenon could be in terms of non linear variations of the opacity sources in the stellar atmospheres in the course of the pulsation of the objects. Hence, such multicolour light curves will form important test cases for dynamic model atmospheres.

\subsubsection{Individual objects}

In the following some notes on individual objects are given:

VY Cas: Several maxima and minima can be found in our dataset. Fourier analysis plus fitting favours a 116 day variation which is comparable to the 100 day GCVS one. Obviously, variability exists also on shorter time scales of several tens of days (the first harmonic of $116 \mathrm{~d}, 5 \mathrm{~d}$, leads to a good fit). A period of 146 days contributes the beating present in the data. 


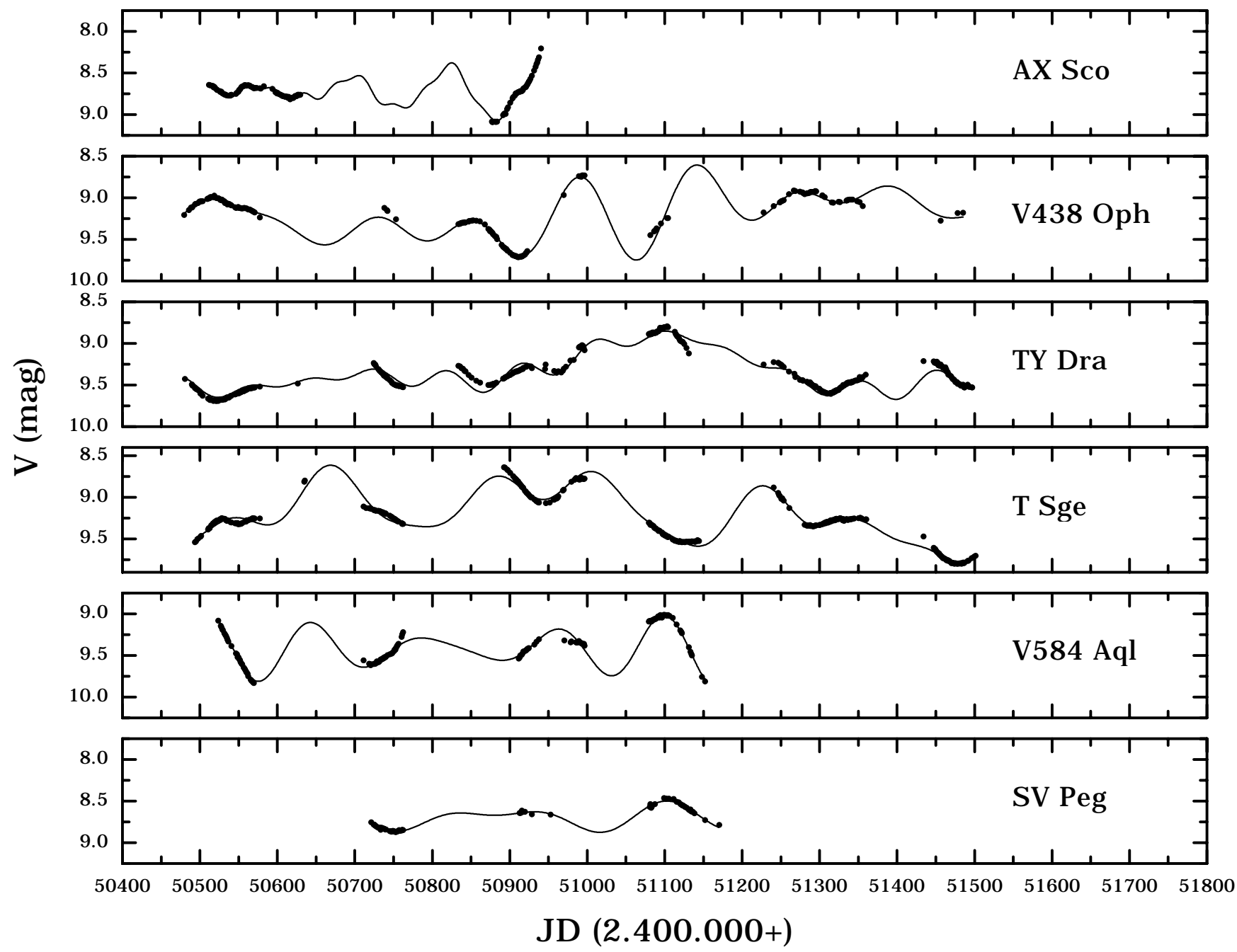

Fig. 2. Compilation of all $V$-light curves, Part 2.

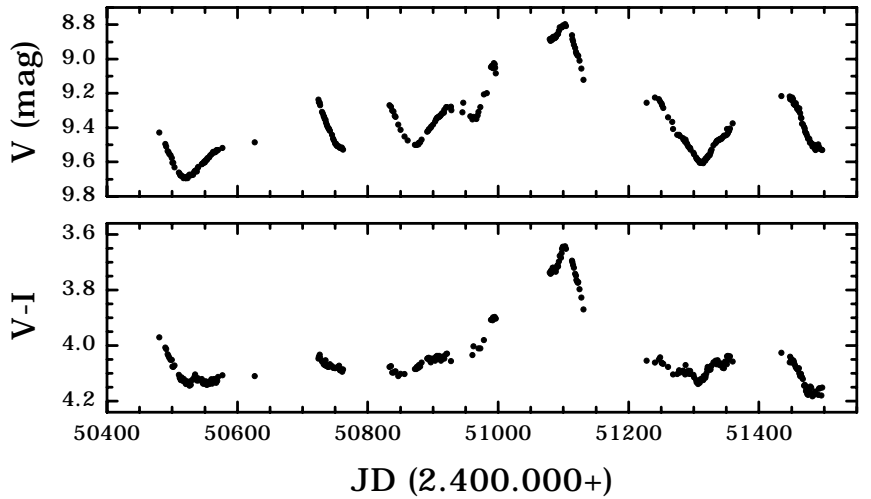

Fig. 3. Comparison of $V$-light curve and $V-I_{\mathrm{C}}$ variation for the Lb variable TY Dra.

A longer modulation of about 800 days seems probable from light curve and Fourier inspection. Houk (1963) also saw a long period of 600 days in her data set. Nevertheless our longer period fits our data much better. An analysis of the Hipparcos data supports the existence of periods in the given ranges including a long-term modulation.
RV Cam: The dataset obtained for this star covers several distinct maxima and minima. However, estimating a mean period is quite difficult as the cycle length varies strongly between 130 and 180 days. There is no combination of two or three periods that can fit the observed light curves. The GCVS period of 101 days is similar to a 109 day variation seen in the data. Strong variations in amplitude as well as a long term variability (about 960 days i.e. the beat period between 177 and $217=2 \times 109$, both also seen in the data) can be observed, too. Finally an additional 132 day period is needed to fit the data set to a still not fully satisfactory extent.

Recently, Koen \& Laney (2000) reported on the discovery of rapid oscillations in M-giants derived from Hipparcos photometry. For the only star in common with us, RV Cam, they find a very short periodicity of only 7.67 days, in addition to the 96.4 day variation from the Hipparcos catalogue. Figure 4 shows both the spectral windows and Fourier amplitude spectra from our APT as well as the Hipparcos data used by Koen \& Laney. 


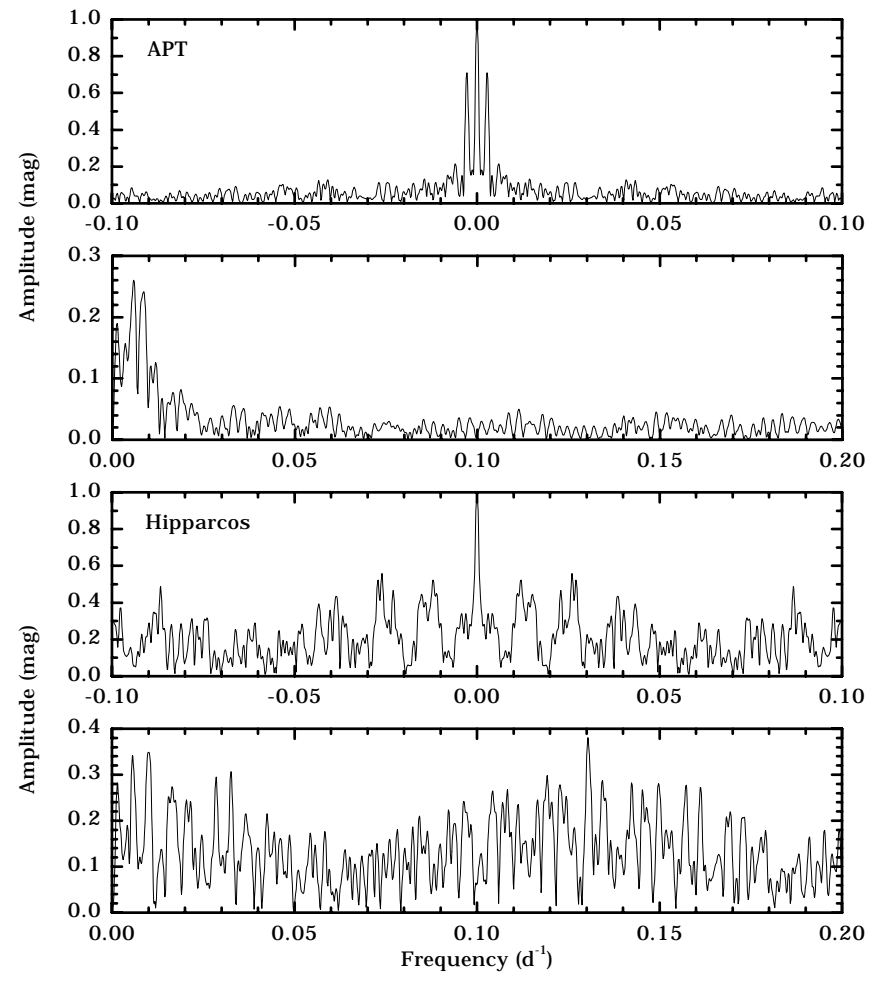

Fig. 4. Spectral windows and fourier amplitude spectra of RV Cam from both APT and Hipparcos data.

Whereas the Hipparcos data set shows the frequency peak at $0.13 \mathrm{~d}^{-1}$ corresponding to the period given by Koen \& Laney, our data set gives no indication of such short time scales, especially at amplitudes of a few tenth of a magnitude. This is surprising, since our data set is larger than the Hipparcos one, has a comparable or higher photometric quality and also a sampling rate that would allow the detection of such short variations (The Nyquist cut-off for our dataset is 2 days!). We will come back to the reality of such rapid variations in Sect. 3.1.4 below.

CW Cnc: The two maxima observed differ by about 70 days in time. The light change is strange with a rise in brightness at the beginning of our dataset that ends in some kind of shoulder before a more "cyclic" variation starts. From this short light curve no certain periods can be derived. Periods of 97 and 100 days combined with their first harmonics at 48.5 and 50 days give the best fit to the data. The Hipparcos material indicates some long term (>1000 days) variation.

RW Vir: The first part of the curve observed for this Lb variable looks quite sinusoidal with an amplitude of about $0.5 \mathrm{mag}$ and a period of about 116 days. The following parts of the light curve present a quite irregular light change with sometimes very low amplitudes. Variations occur on a time scale of about 72 days. The overall modulation has a 1060 day length. The Hipparcos data for RW Vir also show such a long term trend of the order of 1100 days. Wisse \& Wisse (1971) found at least two time scales of variation in their dataset, a small amplitude, short time oscillation and a variation with a larger amplitude on a time scale of at least 100 days. However, the short time variability reported by them is probably due to a variable comparison star (Lebzelter \& Posch 2001). To achieve the poor fit shown in Fig. 1 one has also to include a 201 day period.

Y UMa: Four well-defined maxima can be seen in our light curve of this star. The minima, except one around JD 2450500, are very broad. They may actually represent phases of light constancy. It is therefore difficult to estimate how many maxima of the star may have occurred in the observational gaps in our light curve. The four maxima would suggest a period of about 320 days. The GCVS gives 168 days, i.e. approximately half this period, suggesting further maxima in the gaps. A shorter period is also favoured by the Fourier analysis, which gives 166 days.

The first three light cycles in our dataset show a very similar amplitude of $0.7 \mathrm{mag}$. The fourth light cycle has a much smaller amplitude. On the other hand, the GCVS lists a visual amplitude of 2.1 magnitudes, i.e. three times larger than the current value. There is again variability at longer (725 days) time scales. For the fit shown, 3 independent periods of 331,260 , and 724 as well as 2 harmonics, 165.5 and 362 days, were used.

Recently, Kiss et al. (1999) found three periods (324, 315 , and 164 days) in a study based on international amateur data. Keeping in mind the very different type of data used by them we see a similar behaviour of Y UMa in their monitored five year interval when compared with our material. The Hipparcos data of Y UMa show a 333 day period as well as a $0.13 \mathrm{~d}^{-1}$ frequency peak similar to the RV Cam data (see above). Again no hint of such a short time variation is seen in our data set.

RX Boo: One complete, quite regular light cycle has been observed. A Fourier analysis gives a period of 140 days. For a perfect fit of the somewhat asymmetric light curve, a period of 70 days, the first harmonic, has to be included. The GCVS period of 340 days is not seen in our somewhat short dataset.

Our main periodicity (140 days) is of the same order as the period of 160 days derived from long term monitoring of RX Boo by Taylor (1987). Andronov \& Kudashkina (1988) found two beating periods of 179 and 162 days length. Keping et al. (1984) found a period of only 78 days, close to our first harmonic secondary period of 70 days. Altogether RX Boo seems to have a complex and maybe unstable variability pattern.

g Her: The light curve we obtained for this bright semiregular variable nicely agrees with the GCVS period of 89 days with the cycle length scattering around this value by a few days. Among all SRVs in our APT sample the light change of $\mathrm{g}$ Her is maybe the most regular (except for RX Boo, where we only observed one 
light cycle). A period of about 60 days as suggested by Christian et al. (1995), also from data obtained with an automatic telescope, could not be confirmed. However, as noted above, the period of these stars may change with time. For the fit low amplitude periods of 74 and 103 days were also used.

Beside these short time variations, the APT light curve indicates variability on a much longer time scale of about 1239 days with a similar amplitude as for the short time variation. Such a longer period has been noted already by Houk (1963) and has also been detected in AAVSO data (Kiss et al. 1999). The Hipparcos material of $\mathrm{g}$ Her again has quite rapid variations of 8.6 and 12.4 days as the most prominent fourier peaks, which are not seen in our APT material.

AX Sco: The two parts of the light curve observed do not seem to fit together at all. The first part indicates a low amplitude variability with the two maxima differing by about 90 days. The second part is obviously the rising part of a variation with a larger amplitude of at least one magnitude. The Fourier analysis shows periods in the range 40 to 130 days. The fit shown in Fig. 2 includes 3 independent periods 128, 124, and 39 days as well as the first harmonic of the first one, namely 64 days. One should keep in mind that for such a short dataset no unique solution is possible for this kind of complex light curve.

The Hipparcos period of 127.7 days is also our most important time scale. The Hipparcos material also contains power at shorter time scales.

V438 Oph: The most obvious feature of the light change of this star is the strong variability in mean brightness on a time scale of about 1000 days. Fourier analysis shows a strong 153 day peak and lower amplitude at 129 and 202 days. From a visual inspection we also estimate a time scale of roughly 150 days. The GCVS lists 170 days as period for V438 Oph which is also in the possible range keeping in mind the complex variation patterns of the object. Hipparcos data indicate a strong 149 day period as well as high-power rapid variability at 2.6 and 4.0 days (see below).

TY Dra: As for V438 Oph, the mean brightness of the Lb TY Dra varies significantly at a long (820 days) time scale. Variations with periods of 89 and 107 days are present. The fit shown also includes the first harmonic of 820 days, namely 410 days.

T Sge: The star shows its main variability on a time scale of 178 days, similar to the 166 days period listed by the GCVS. The mean brightness is highly variable which may indicate a second, significantly longer, 1100 day period. Fourier analysis suggests additional amplitudes at 112 and 316 days.

V584 Aql: Four parts of light cycles have been observed but only one distinct maximum and one minimum was covered. The two descending branches of the light curve observed at the begin and at the end of our dataset are parallel and can be used to estimate a time scale of the variation. We estimate that there are three light cycles in between these two branches, giving a period of approximately 150 days. One would expect a further maximum somewhere around JD 2450950 which would be in agreement with the period derived. Although the star has been classified as Lb in the GCVS, deriving one period seems to be reasonable for the time the star was monitored. However, a strong variability in the amplitude is clearly visible. This can be fitted by two beating periods with 143 and 123 days length.

SV Peg: Only very short parts of the light curve have been monitored. They do not allow us to derive a certain period. The GCVS period of 145 days agrees with our findings. A good fit to the data can be obtained by a combination of a 145 day period with a longer, 220 day variation. Power in the Hipparcos material is found at 170 days as well as at again quite high frequencies corresponding to a 9 day period.

\subsubsection{Rapid oscillations?}

As stated above, the results of Koen \& Laney (2000) support the presence of rapid oscillations in M-giants derived from Hipparcos photometry. For the only star in common with us, RV Cam, we already showed that no comparable short period is present in our APT material. An inspection of the available Hipparcos photometry for the other stars of our sample shows such short periods (between 3 and 12 days, clustering around 7 days) in 8 out of 13 objects! Typically these periods have amplitudes of a few tenths of a magnitude and are often the strongest peaks in the Fourier plots! None of our APT data show such peaks at high frequencies although the Nyquist cut-off of our material at about 2 days would easily allow such detections.

Only two interpretations seem possible: either the short-term variations are intrinsic but of a transient nature (for all objects?) or we see an instrumental effect (see Sect. 4) in the Hipparcos data which would be of a surprisingly high magnitude. Only APT-monitoring of some of the other objects in Koen \& Laney, maybe at an even higher time resolution, can clarify this but the reality of such rapid variations, at least with the quoted amplitudes, seems very questionable and in contradiction with the new observational material available to us.

\subsection{Visual phases of Miras from AAVSO data}

For the Miras in our sample no APT observations have been obtained. To set the infrared photometry (see below) in relation with the visual light change we derived phases from AAVSO data (Mattei, private communication, and Mattei 1998, 1999, 2000). As the cycle length of Miras typically varies by a few days, too, we used the maximum actually preceding each infrared data point and the current period resulting from the following maximum to derive the phase. Phases are listed in Col. 4 of Tables B.2 to B.5. 


\subsection{Multi-epoch infrared photometry}

The phase coverage of our NIR-measurements is not comparable with the well-sampled light curves in the visual (see APT-section above). It is better to speak of multiepoch photometry in most of the cases. It can be used to estimate a better average luminosity or to get at least a lower limit for the pulsational amplitudes in the infrared. Also more typical mean near infrared colours can be derived.

\subsection{Light curve shapes and phase differences}

Only the oxygen-rich Mira R Cas and the Carbon-Mira T Dra have something that could be called a "light curve" (see Fig. 5).

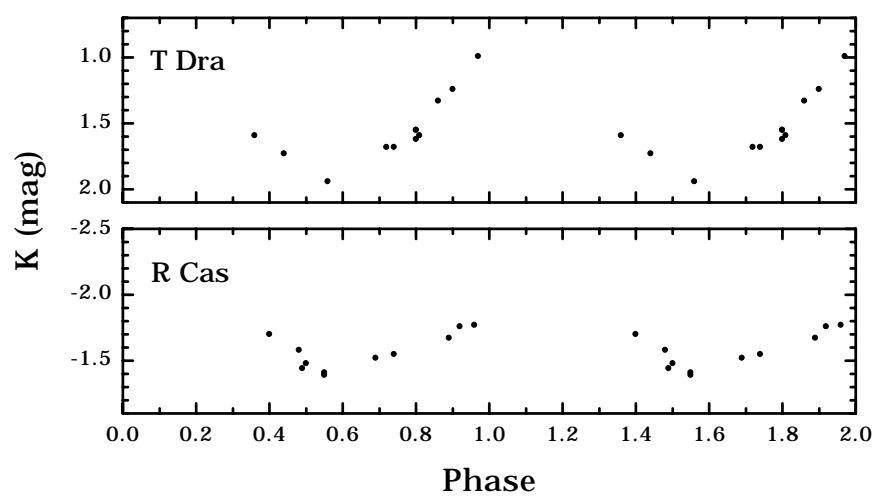

Fig. 5. $K$-filter phase diagramms of the Carbon-Mira T Dra and the Oxygen-rich Mira R Cas. Points up to phase 2.0 are repeated for clarity.

The NIR-light curves and the corresponding, visual AAVSO maxima and minima allow a check for potential phase shifts between the two wavelength ranges. Unfortunately the asymmetry of the respective light curves adds some uncertainty here. From the AAVSO data it is evident that the minima of both $\mathrm{R}$ Cas and $\mathrm{T}$ Dra happen after the mean between two following maxima, which means the rising part of the visual light curve is steeper than the descending one. This delay amounts to 0.1 and 0.06 in phase, respectively. Generally, the smaller amplitude NIR-light curves seem to be more symmetric, at least within the normal uncertainties (see also Quercy 1986). Consequently, a normal sine was fitted through the NIR (K) data with the visual period of that epoch fixed (434 days for R Cas and 430 days for T Dra).

From these fits, phase differences with respect to the AAVSO maxima and minima could be derived. The adoption of a mean phase shift avoids problems with the asymmetries. The resulting values agree for $\mathrm{R}$ Cas and $\mathrm{T}$ Dra and amount to a phase lag of 0.06 for the $K$ curves with respect to the visual ones.

This result is consistent with the view that $K$ represents quite well the true luminosity change, which is known to be more symmetric and somewhat delayed with respect to the more asymmetric visual light curve (compare with Quercy 1986).

\subsection{Near infrared colours}

In order to give an overview of the near infrared properties of the sample, Fig. 6 displays a two colour diagram of all O-rich variables with averaged values for objects with multi-epoch photometry.

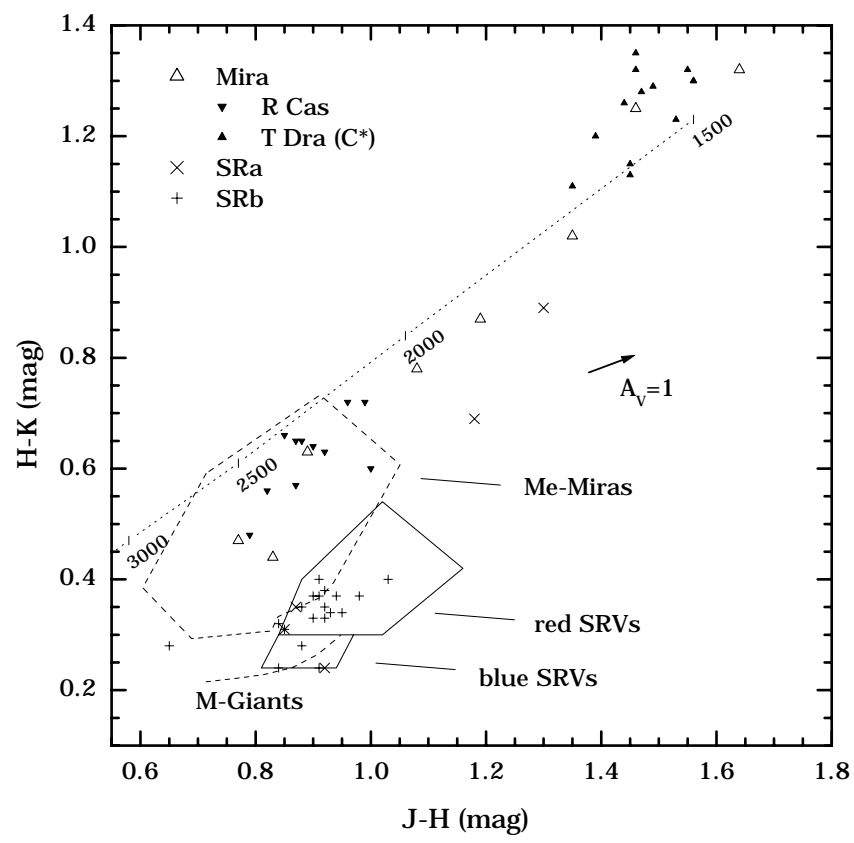

Fig. 6. Near infrared two colour diagram of O-rich variables.

The regions where blue and red SRVs are found are indicated by full lines (from Kerschbaum et al. 1996). The dashed box is the area occupied by Me-Miras taken from Feast et al. (1982). The curved, dashed line is the mean locus of non-variable M-giants in the solar neighbourhood (Feast et al. 1990). The colours of Me-Miras and M-giants were transformed from the SAAO to the ESO photometric system using the results of Kerschbaum \& Hron (1994). The colours for blackbodies of different temperatures $[\mathrm{K}]$ are also indicated for reference. A reddening vector displays the colour shift for $A_{V}=1$.

The observed C-rich Lb variables were not included in the plot since they extend towards much redder colours (up to 2.5 in both indices). An exception is the C-Mira $\mathrm{T}$ Dra, were we plotted all individual measurements in order to see the colour variability. The same was done for the O-rich Mira R Cas.

\section{Discussion}

All our sample objects are now characterised by their pulsational properties in order to make better use of our ISO spectra. The derived quantities (i.e. periods, amplitudes, regularity) verify that our ISO sample covers nearly the 
full range of variability on the AGB. A comparison with data from the literature (e.g. the GCVS) demonstrates again the need for a monitoring approach. Single epoch material is only of very limited use. Moreover, keeping in mind the unpredictable long term variability of the SRVs and IRVs, the quasi-simultaneous observation of the ISO spectra and our photometric time series was very important. Only for Miras can AAVSO data provide this the smaller amplitudes of the SRVs and IRVs limit the use of purely visually-collected data (Lebzelter \& Kiss, in preparation).

For two Miras, R Cas and T Dra, we could estimate a phase lag of $6 \%$ of the near infrared curve with respect to the more asymmetric visual one. The mean near infrared colours of our sample objects are typical for the respective groups. A few Miras and SRVs are seen beyond the region of Me-Miras defined by Feast et al. (1982) in Fig. 6. These objects are obviously highly reddened due to a large amount of circumstellar material.

As well as the characterisation of the stars observed with ISO the presented data allow for some further conclusions on the light change of semiregular and irregular variables. We did not find any difference between these two groups of variables concerning the periodicity of their light change. If there is a true difference between these two variability classes it is obviously only detectable at considerably longer time scales.

For most variables of our sample it turned out to be impossible to describe the visual light change properly over a long time with the coupling of two or three excited periods. This explanation for semiregular behaviour has been suggested before by several authors (see Querci 1986). While we cannot exclude that an interpretation of the light change by the coupling of two periods may be correct for selected SRVs, we think that our investigation has outlined three severe problems in this context: On the one hand, we found that the light change can be nicely fit with a few periods over a relatively short time scale of a few cycles. Keeping in mind the typical length of photometric data sets on these stars published (compare Lebzelter et al. 1995), this interpretation requires in each case a detailed check with a long time base photometric dataset. On the other hand, previous investigations based on long time series are typically based on data from amateur astronomers (e.g. Mattei et al. 1997). The disadvantage of these data is their lower photometric accuracy. Therefore small amplitude variations present in the light curves presented in this paper may be smoothed and missed by the fitting. Finally, in cases where other photometric data sets give different values for the period, we may be confronted with a change of the period on relatively short time scales.

The Fourier amplitude spectrum would allow us to select further possible periods, and this may well improve the fit of the light curve. However, the problem is to attribute a physical meaning to such a large number of periods. While the longest periods found are very unlikely due to pulsation (e.g. Wood 2000), the shorter periods possibly represent different pulsation modes. Models of the atmospheres of these stars would need to explain such a multiple excitation of modes.

The APT data we present here are of sufficient accuracy and time resolution to detect even small amplitude variability on time scales as short as a few days. We could therefore search for such short-term variations which have been detected in red giants from Hipparcos data (Koen \& Laney 2000). These rapid oscillations have not been found in any of the stars in our sample, although they are present in a large fraction of the Hipparcos data of these stars. We think that the most likely explanation for this peak in the Hipparcos data at short periods is an instrumental effect.

Such an effect may be related to the colour correction applied in the reduction of the Hipparcos data, which is somewhat more difficult for red giant stars due to the lack of standards with large $V-I_{\mathrm{C}}$ values. According to van Leeuwen et al. (1997) the colour terms in the transformation of the individual Hipparcos measurements into photometric data are only well defined up to $V-I_{\mathrm{C}}=2.2 \mathrm{mag}$ with a more uncertain linear extrapolation to values of $V-I_{\mathrm{C}}=3 \mathrm{mag}$. The critical components could be the mixed terms containing both the colour and the position in the field of the object at the epoch of the individual observation.

All our program stars have significantly larger $V-I_{\mathrm{C}}$ values (with mean values ranging from 3.5 to $4.6 \mathrm{mag}$ ). $75 \%$ of Koen \& Laney's (2000) objects also have $V-I_{\mathrm{C}}$ values larger than $2.2 \mathrm{mag}$ and $10 \%$ are even above 3 mag. Since 8 of our 13 objects with Hipparcos data show short time variations therein but not in our APT measurements one has to doubt the reality of similar variations in red objects like those discussed by Koen \& Laney (2000). It would be interesting to check some of them by independent ground-based time series.

A colour-dependent effect would be in agreement with the fact that such variations have not been reported for stars with earlier spectral types, like Cepheids, for which they obviously would have been noted. Naturally, a more detailed investigation is needed to clarify the origin of these variations. This is far beyond the scope of this paper.

Finally, we want to briefly comment on the observed difference between $V$ light curve and $V-I_{\mathrm{C}}$ colour variation. A radial pulsation would suggest that the ratio between the light change and the colour change is given by a constant function for a given star. We observe some phases that are accompanied by strong colour changes while other phases show almost no variability in colour. If we accept the $V-I_{\mathrm{C}}$ value as some kind of temperature indicator this would mean that some of the light change observed is happening at an almost constant overall temperature of the star. This suggests that beside radial pulsation other mechanisms are contributing to the light change in these objects as well.

\section{Future prospects}

It was the aim of this paper to present new highquality light curves of SRVs and IRVs and to discuss the 
individual aspects of the light change found in these stars with the help of a small sample of objects especially selected to complement our spectroscopic ISO observations. Observations of semiregular variables with the Austrian APT will continue. Currently two other AGB-star observing programs are in progress. The sample of the first one consists of 28 short-period SRVs, the second one of 14 irregular variables. In both programs the stars are monitored with the same accuracy and time resolution as in this paper. The complexity of the light change of SRVs and IRVs demands a detailed and extensive discussion of individual objects, which makes it necessary to present the results in several papers.

As soon as the whole sample has been reduced we plan to make all visual light curve measurements of our program available to the astronomical community via the internet. Moreover, such a larger sample of SRVs and IRVs will hopefully provide important clues about the interrelation of these two variability classes. As noted by Lebzelter et al. (1995) and Kerschbaum et al. (1996) the classification of stars as irregular variables, i.e. with no derivable period, might not be correct for a number of objects. Moreover the distinction between SRb and Lb variability is impossible in most of the cases and mainly unphysically determined by the quality of the available observational material.

Acknowledgements. The authors are grateful to the anonymous referee for providing valuable input helping to improve the scientific content of the paper.

The work of F. K. was supported by APART (Austrian Programme for Advanced Research and Technology) from the Austrian Academy of Sciences and the Oesterreichische Nationalbank under the Jubiläumsfonds-project number 6876 . This research and especially the APT was funded by the Fonds zur Förderung der wissenschaftlichen Forschung under project number S7308-AST, S7301-AST and P14365-PHY.

We wish to thank Thomas Granzer and Mike Seeds for their support in the use of the APTs.

The authors are very grateful to the TCS staff for carefully performing the service observations. Some observing runs at TCS where supported by the European Commission through the TMR Programme (Access to Large-Scale Facilities).

This research has made use of the SIMBAD database. In this research we have used, and acknowledge with thanks, data from the AAVSO International Database, based on observations submitted to the AAVSO by variable star observers world wide.

\section{Appendix A: APT comparison stars}

Table A.1 lists $V$ mean magnitudes and $V-I_{\mathrm{C}}$ colours for the comparison stars used in this paper. The number of absolute flux measurements used to derive the actual magnitudes and the scatter of these measurements are given as well. The difference between our $V$ values and values published in the literature (typically from the Hipparcos mission) is below 0 m $04^{\mathrm{m}}$ in all cases. No systematic difference has been found.
Table A.1. Photometric data of APT comparison stars. The last column gives the number of measurements used to derive the $V$ and $V-I_{\mathrm{C}}$ values.

\begin{tabular}{lccrl}
\hline $\begin{array}{l}\text { Comp. } \\
\text { star }\end{array}$ & $\begin{array}{c}V \\
{[\mathrm{mag}]}\end{array}$ & $\begin{array}{c}V-I_{\mathrm{C}} \\
{[\mathrm{mag}]}\end{array}$ & $\begin{array}{r}\text { numb. } \\
\text { meas. }\end{array}$ & for \\
\hline HD4811 & $7.96 \pm 0.04$ & $1.64 \pm 0.05$ & 167 & VY Cas \\
HD237271 & $8.59 \pm 0.04$ & $1.62 \pm 0.04$ & 219 & RV Cam \\
HD78279 & $9.43 \pm 0.03$ & $0.96 \pm 0.02$ & 40 & CW Cnc \\
HD105061 & $7.62 \pm 0.05$ & $1.1 \pm 0.03$ & 284 & RW Vir \\
HD110463 & $8.26 \pm 0.04$ & $1.07 \pm 0.04$ & 248 & Y UMa \\
HD126598 & $7.41 \pm 0.03$ & $1.31 \pm 0.03$ & 46 & RX Boo \\
HD148434 & $6.97 \pm 0.05$ & $1.16 \pm 0.04$ & 174 & g Her \\
HD150346 & $8.87 \pm 0.05$ & $1.37 \pm 0.03$ & 46 & AX Sco \\
HD155768 & $9.33 \pm 0.05$ & $0.75 \pm 0.04$ & 100 & V438 Oph \\
HD238719 & $9.66 \pm 0.05$ & $1.03 \pm 0.05$ & 273 & TY Dra \\
HD231206 & $8.87 \pm 0.05$ & $0.67 \pm 0.04$ & 256 & T Sge \\
HD191316 & $8.79 \pm 0.05$ & $1.23 \pm 0.06$ & 87 & V584 Aql \\
HD210417 & $8.07 \pm 0.04$ & $1.70 \pm 0.05$ & 55 & SV Peg \\
\hline
\end{tabular}

\section{Appendix B: Near infrared data}

Tables B.2 to B.5 list all near-infrared photometry of AGB-stars presented in this paper.

Besides GCVS4- and IRAS-name, variability type, chemistry (O-rich or C-rich), the Julian day number and a code "pub." denoting the reference of literature data given. In case of Miras also an AAVSO-phase (see above) is given for reference.

Estimates of the errors of the NIR-photometry taken are given in Table B.1. The given values are calculated from the individual programme- and standard-starmeasurements. The errors do not include uncertainties of the photometric system.

Table B.1. Typical total errors of NIR-photometry.

\begin{tabular}{cccccc}
\hline$c$ & $\mathrm{Mag}$ & $\sigma_{\mathrm{J}}$ & $\sigma_{H}$ & $\sigma_{K}$ & $\sigma_{L^{\prime}}$ \\
\hline $\mathrm{I}$ & $<3$ & 0.03 & 0.03 & 0.03 & $0 .{ }^{\mathrm{m}} 03$ \\
& 4 & $\cdot$ & $\cdot$ & & \\
& 5 & 0.04 & & & \\
\hline
\end{tabular}

\section{References}

Andronov, I. L., \& Kudashkina, L. S. 1988, Astron. Nachr., 309, 323

Aringer, B., Kerschbaum, F., Hron, J., et al. 1999, Proc.: Asymptotic Giant Branch Stars, ed. T. Le Bertre, A. Lèbre, \& C. Waelkens, ASP, IAU Symp., 191, 169

Bagnulo, S. 1996, Ph.D. dissertation, Queen's University Belfast, N. Ireland

Boyd, L. J., Genet, R. M., \& Hall, D. S. 1984, IBVS, 2511

Catchpole, R. M., Robertson, B. S. C., Lloyd-Evans, T. H. H., et al. 1979, Circulars. South African Astron. Obs. V., 1, 61

Christian, V. C., Donahue, R. A., Soon, W. H., et al. 1995, PASP, 107, 411 
Feast, M. W., Robertson, B. S. C., Catchpole, R. M., et al. 1982, MNRAS, 201, 439

Feast, M. W., Whitelock, P. A., \& Carter, B. S. 1990, MNRAS, 247,227

Hinkle, K. H. 1978, ApJ, 220, 210

Höfner, S. 1999, A\&A, 346, L9

Höfner, S., Jørgensen, U. G., Loidl, R., \& Aringer, B. 1998, A\&A, 340, 497

Houk, N. 1963, AJ, 68, 253

Hron, J., Loidl, R., Höfner, S., et al. 1998, A\&A, 335, L69

Jørgensen, U. G., Hron, J., \& Loidl, R. 2000, A\&A, 356, 253

Keping, Z., Yunxiang, H., Peisheng, C., Yun, Z., \& Heng, G. 1984, Ap\&SS, 107, 373

Kerschbaum, F. 1995, A\&AS, 113, 441 (SRIIb)

Kerschbaum, F., \& Hron, J. 1994, A\&AS, 106, 397 (SRIIa)

Kerschbaum, F., Lazaro, C., \& Habison, P. 1996, A\&AS, 118, 397 (LBI)

Kiss, L. L., Szatmary, K., Cadmus, R. R., \& Mattei, J. A. 1999, A\&A, 346, 542

Kiss, L. L., Szatmary, K., Szabo, Gy., \& Mattei, J. A. 2000, A\&AS, 145, 283

Koen, C., \& Laney, D. 2000, MNRAS, 311, 636

Koornneef, J. 1983a, A\&AS, 51, 489

Koornneef, J. 1983b, A\&A, 128, 84

Le Bertre, T. 1992, A\&AS, 94, 377L

Lebzelter, T. 1999, A\&A, 351, 644

Lebzelter, T., \& Posch, T. 2001, IBVS, 5089

Lebzelter, T., Kerschbaum, F., \& Hron, J. 1995, A\&A, 298, 159

Loumos, G. L., \& Deeming, T. J. 1978, Ap\&SS, 56, 285
Mattei, J. A. 1998, AAVSO Bulletin, 61

Mattei, J. A. 1999, AAVSO Bulletin, 62

Mattei, J. A. 2000, AAVSO Bulletin, 63

Mattei, J. A., Foster, G., Hurwitz, L. A., et al. 1997, Proc. of the ESA Symposium Hipparcos Venice '97, ed. B. Battrick, ESA SP-402, 269

Percy, J. R., \& Au, W. 1994, IBVS, 4114

Percy, J. R., Desjardins, A., Yu, L., \& Landis, H. J. 1996, PASP, 108, 139

Posch, T., Kerschbaum, F., Mutschke, H., et al. 1999, A\&A, 352,609

Querci, F. R. 1986, in The M-type stars, ed. H. R. Johnson, \& F. R. Querci, NASA SP-492, 1

Sperl, M. 1998, Comm. Asteroseismology (Vienna), 111, 1

Strassmeier, K. G., Boyd, L. J., Epand, D. H., \& Granzer, T. 1997, PASP, 109, 697

Taylor, M. D. 1987, JBAA, 97, 277

van Belle, G. T., Dyck, H. M., Benson, J. A., \& Lacasse, M. G. 1996, AJ, 112, 2147

van Leeuwen, F., Lindegren, L., \& Mignard, F. 1997, The Hipparcos and Tycho Catalogues, vol. 3, ESA SP-1200

Whitelock, P. A., Feast, M. W., Marang, F., \& Overbeek, M. D. 1997, MNRAS, 288, 512

Whitelock, P. A., Marang, F., \& Feast, M. W. 2000, MNRAS, 319, 728

Winters, J. M., Keady, J. J., Gauger, A., \& Sada, P. V. 2000, A\&A, 359, 651

Wisse, M., \& Wisse, P. N. J. 1971, A\&A, 12, 143

Wood, P. R., 2000, Publ. Astron. Soc. Aust., 17, 18 